\title{
Dither for Smoothing Relay Feedback Systems
}

\author{
Luigi Iannelli, Member, IEEE, Karl Henrik Johansson, Member, IEEE, Ulf T. Jönsson, Member, IEEE, and \\ Francesco Vasca, Member, IEEE
}

\begin{abstract}
Dither signals provide an effective way to compensate for nonlinearities in control systems. The seminal works by Zames and Shneydor, and more recently, by Mossaheb, present rigorous tools for systematic design of dithered systems. Their results rely, however, on a Lipschitz assumption relating to nonlinearity, and thus, do not cover important applications with discontinuities. This paper presents initial results on how to analyze and design dither in nonsmooth systems. In particular, it is shown that a dithered relay feedback system can be approximated by a smoothed system. Guidelines are given for tuning the amplitude and the period time of the dither signal, in order to stabilize the nonsmooth system.
\end{abstract}

Index Terms-Averaging theory, bound error estimation, dither, limit cycles, linear matrix inequalities (LMIs), nonsmooth systems, practical stability, relay feedback.

\section{INTRODUCTION}

$\mathbf{T}$ HE use of dither signals for stabilization of nonlinear control systems is a well-known and frequently used technique. The idea is that by injecting a suitably chosen high-frequency signal in the control loop, the nonlinear sector is effectively narrowed and the system can thereby be stabilized. Theoretical justification of this idea for systems with continuous nonlinearities has been obtained by Zames and Shneydor [1], [2] and Mossaheb [3]. Their results rely however, on a crucial Lipschitz assumption on the nonlinearity, and thus, do not cover important applications with discontinuities. Indeed, discontinuous nonlinearities in feedback-control systems with high-frequency excitations appear in a large variety of applications, including systems with adaptive control [4], friction [5], [6], power electronics [7], pulsewidth modulated converters [8], quantizers [9], relays [10], and variable-structure controllers [11]. In their paper on the analysis of the (smooth) LuGre friction model, Pervozvanski and Canudas de Wit [12] pointed out that a rigorous analysis of dither in discontinuous systems does not exist. Dither tuning of general nonsmooth systems is, to our knowledge, limited to approximate design methods mainly based on describing functions [13], [14]. In power electronic systems such as various

Manuscript received September 9, 2002; revised April 14, 2003. The work by L. Iannelli and F. Vasca was supported by the European Commission within the SICONOS project. The work by K. H. Johansson and U. Jönsson was supported by the Swedish Research Council and by the European Commission within the RECSYS project. This paper was recommended by Guest Editor C. K. Tse.

L. Iannelli is with the Department of Computer and Systems Engineering, University of Napoli "Federico II," 80125 Naples, Italy (e-mail: luiannel@unina.it).

K. H. Johansson is with the Department of Signals, Sensors and Systems, Royal Institute of Technology, 10044 Stockholm, Sweden (e-mail kallej@s3.kth.se).

U. T. Jönsson is with the Department of Mathematics, Royal Institute of Technology, 10044 Stockholm, Sweden (e-mail: ulfj@math.kth.se).

F. Vasca is with the Department of Engineering, University of Sannio in Benevento, 82100 Benevento, Italy (e-mail: vasca@ unisannio.it).

Digital Object Identifier 10.1109/TCSI.2003.815194 types of dc-dc converters, averaging theory is applied to separate the slow dynamics from the fast dynamics, for example, imposed by switching elements in pulsewidth modulation. Rigorous averaging analysis have been done for this class of nonsmooth systems [7], [15].

The contribution of this paper is a theory for the design of dither in nonsmooth feedback systems. We limit the analysis to an important class of nonlinearities, namely, relays. The reason for this is that these systems are common. Early motivation for studying relay systems come from mechanical and electromechanical systems [16], [10]. Recently, there has been renewed interest due to a variety of emerging applications, such as automatic tuning of proportional integral derivative (PID) controllers [17], quantized control [18], and supervisory control [19]. The analysis of relay feedback systems is nontrivial, even if the dynamical part of the system is linear. Major progress in the study of various properties of autonomous linear systems with relay feedback was achieved in the last decade, particularly in the understanding of limit cycles in these systems, e.g., [20]-[25]. See [10] and [21] for further historical remarks and references on relay feedback systems.

In our study of the discontinuous dithered system, we adopt an averaging approach, which is a widely applied mathematical tool. The main observation is that the nonlinearity $n(z)$ of the dithered system can be approximated by the smoothed nonlinearity $N(z)=p^{-1} \int_{0}^{p} n(z+\delta(t)) d t$, where $\delta(t)$ is the dither signal and $p$ its period time. Instead of studying a nonlinear system with an external high-frequency signal $\delta(t)$, one can study a smoothed system with a "nicer" nonlinearity $N(z)$. In the paper, the nonlinearity $n(z)$ of the dithered system is a relay (sign function) and the dither $\delta(t)$ is a triangular signal. This leads to that the smoothed nonlinearity $N(z)=\operatorname{sat}(z / A)$, where sat denotes the saturation function and $A$ is the amplitude of the dither signal. A saturated system is, in general, easier to analyze than a dithered relay system. In this paper, it is shown how to relate the behavior of the smoothed system in a precise way to the behavior of the dithered system. We show that the dither period determines the accuracy of this approximation: the smaller the dither period $p$, the closer is the trajectory of the dithered system to the trajectory of the smoothed system. Since the dither amplitude $A$ determines the gain of the saturation, and thus the stability of the smoothed system, stability of the smoothed system is linked to (practical) stability of the dithered system. Hence, by using existing stability results for saturated systems (such as the Zames and Falb criterion), we can draw stability conclusions on the dithered system. These results are collected into a design procedure for dithered systems, where the dither signal is adjusted to the dynamics of the linear part of the system.

In this paper, the behavior of the dithered relay feedback system is shown to be highly affected by the shape of the dither 
signal. This feature is interesting since relay feedback systems are known to exhibit several complex behaviors, such as quasiperiodic orbits [26], chattering and sliding periodic orbits [21], [25], and asymmetric orbits [22]. When the dither signal is a square wave, the dithered system can exhibit an asymmetric periodic orbit, though the smoothed system is asymptotically stable. We even show an example in which, by using a trapezoidal dither signal, both systems have a stable oscillation, but the period time for the oscillation of the smoothed system is different from the one of the dithered system. It seems that dither signals with zero slope over nonzero time intervals lead to less predictable systems. This is in stark contrast to systems with Lipschitz continuous dynamics for which it can be shown that the form of the dither signal is not critical at all, see [1] and [2].

The outline of the paper is as follows. The preliminaries are presented in Section II. The main results are given in Section III showing that the solutions of the dithered system can be arbitrarily well approximated by the solutions of a smoothed system. The section also discusses practical stability. Section IV relates these results to dither design. Our main design conditions can be formulated in terms of linear matrix inequalities (LMIs). A brief discussion on relay feedback systems with other dithers is given in Section V. The paper is concluded in Section VI.

\section{Dithered Relay FeEdback Systems}

The particular class of nonsmooth dithered system considered in this paper is the linear system with relay feedback. This section presents the notation and a motivating example.

\section{A. Dithered System}

The dithered system is the relay feedback system

$$
\dot{x}(t)=L x(t)+b n(c x(t)+r(t)+\delta(t)), \quad x(0)=x_{0} .
$$

Here, $L, b$, and $c$ are constant matrices of dimensions $q \times q$, $q \times 1$, and $1 \times q$, respectively, where $q \geq 1$. The nonlinearity $n: \mathbb{R} \rightarrow \mathbb{R}$ is given by the relay characteristic

$$
n(z)=\operatorname{sgn}(z)= \begin{cases}1, & z>0 \\ 0, & z=0 \\ -1, & z<0 .\end{cases}
$$

The reference signal $r(t)$ is assumed to be Lipschitz continuous, i.e., there exists a constant $M_{r}>0$ such that $\left|r\left(t_{1}\right)-r\left(t_{2}\right)\right| \leq$ $M_{r}\left|t_{1}-t_{2}\right| \forall t_{1}, t_{2} \geq 0$.

The dither signal $\delta:[0, \infty) \rightarrow \mathbb{R}$ is periodic and of high frequency compared to the linear dynamics. An example of a dither signal, which we will study in detail, is a triangular waveform of amplitude $A>0$ and period $p>0$, i.e., $\delta(t+p)=\delta(t)$ for all $t$ and

$$
\delta(t)= \begin{cases}\frac{4 A}{p} t, & t \in\left[0, \frac{p}{4}\right) \\ -\frac{4 A}{p} t+2 A, & t \in\left[\frac{p}{4}, \frac{3 p}{4}\right) \\ \frac{4 A}{p} t-4 A, & t \in\left[\frac{3 p}{4}, p\right) .\end{cases}
$$

It should be pointed out that the results in this paper depend on the shape of the dither signal. Dither signals with zero slope for nonvanishing time intervals, such as the square wave, are sometimes unpredictable. This is in contrast to systems with Lipschitz continuous dynamics, where the form of the dither signal is not critical [1], [2]. Throughout the paper, we will consider triangular dither and, when needed, we will highlight differences due to the use of other dither signals.

The relay feedback system is assumed to have a solution $x$ : $[0, \infty) \rightarrow \mathbb{R}^{n}$ (in a classical sense), which, on every compact subinterval of $[0, \infty)$ is $C^{1}$ everywhere except at finitely many points. In general, the solution must not be unique. This is not of interest here, since the results in the paper hold for any of these solutions. We sometimes use the notation $x\left(t, x_{0}\right)$ for the solution of (1). We use $|\cdot|$ to denote the Euclidean norm of a vector and $\|\cdot\|$ to denote the corresponding induced matrix norm. The notation $X>0(X \geq 0)$ is used to denote that a matrix $X$ is positive (semi) definite.

\section{B. Smoothed System}

The smoothed system is defined as

$$
\dot{w}(t)=L w(t)+b N(c w(t)+r(t)), \quad w(0)=w_{0}
$$

where the smoothed nonlinearity $N: \mathbb{R} \rightarrow \mathbb{R}$ is the average $N(z)=p^{-1} \int_{0}^{p} n(z+\delta(t)) d t$. For the triangular dither, it is easy to show that

$$
\begin{aligned}
N(z) & =p^{-1} \int_{0}^{p} \operatorname{sgn}(z+\delta(t)) d t \\
& =\operatorname{sat}\left(\frac{z}{A}\right)= \begin{cases}1, & z>A \\
\frac{z}{A}, & |z| \leq A \\
-1, & z<-A .\end{cases}
\end{aligned}
$$

It will be shown below that the smoothed system in many cases is a good approximation of the dithered relay feedback system. Therefore, analysis and design can be performed on the smoothed system, which is often easier to treat, and then be carried over to the dithered system.

Note that the term "smoothed system" (which is standard in the literature on dither) refers to the fact that the nonlinear sector is narrowed by the dither signal. The nonlinearity is not necessarily $C^{\infty}$, as illustrated above by the saturation function.

\section{A Motivating Example}

A second-order relay feedback system is used as a representative example. Consider the system (1) with $r \equiv 0$ and

$$
L=\left[\begin{array}{cc}
-2 & -1 \\
1 & 0
\end{array}\right] \quad b=\left[\begin{array}{l}
1 \\
0
\end{array}\right] \quad c=\left[\begin{array}{ll}
1 & -1
\end{array}\right]
$$

When no dither is present $(\delta(t) \equiv 0)$, the relay feedback system presents a limit cycle as reported in Fig. 1(a). The output of the linear part $-c x$ of (1) is plotted for a solution with initial condition $x_{0}=\left[\begin{array}{ll}2 & 1\end{array}\right]^{T}$. If we apply a triangular dither signal with amplitude $A=1$ and period $p=1$, the limit cycle in Fig. 1(a) is reduced as shown in Fig. 1(b). Hence, the dither in a sense attenuates the oscillations present in the original system. Fig. 1(b) also shows the output $-c w$ of the smoothed system (3). The two systems have different responses. If we decrease the dither period $p$ (e.g., $p=1 / 50$ ) the smoothed system and the original dithered system have practically identical outputs. Hence, the smoothed system provides an accurate approximation of the dithered system for $p=1 / 50$. This suggests that the dither period $p$ is related to how accurately the smoothed system approximates the dithered system. In Section III, it is shown 


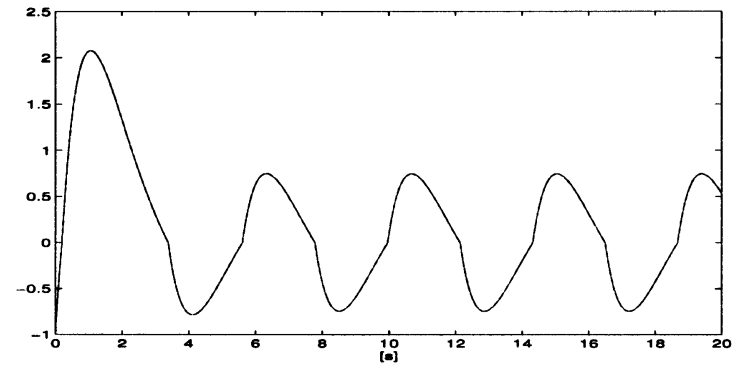

(a)

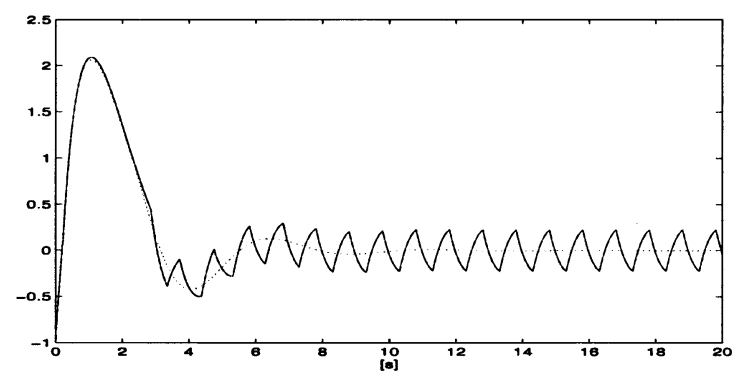

(b)

Fig. 1. Outputs $-c x$ of the dithered relay feedback system (solid) and the smoothed system (dashed) with triangular dither. (a) $A=0$ and $p=1 / 50$. (b) $A=1$ and $p=1 / 50$

that by choosing $p$ sufficiently small, the approximation can be made arbitrarily tight (Theorem III.1). Regarding the dither amplitude $A$, note that the smoothed system above is unstable for $0<A<1 / 2$, since the closed-loop system is linear with characteristic polynomial equal to $s^{2}+\left(2-A^{-1}\right) s+1+A^{-1}$ when $|c w|<A$. The dither amplitude hence defines the response dynamics. This is shown in the next section by relating $A$ to the stability of the dithered system (Theorem III.2).

\section{ANALYSIS}

This section presents results for the dithered system in (1) with the triangular dither signal in (2). The first result is on accurate approximation over compact time intervals and the second is on practical stability. These two results are then combined to obtain a result on approximation over infinite time horizon. The proofs do not fully exploit the particular structure of the smoothed system in (3) and the resulting bounds on the dither period are conservative. In Theorem III.4, we obtain much tighter bounds by using LMIs to characterize the structural properties of the system.

\section{A. Averaging Theorem}

The following theorem states that by choosing the dither period $p$ of the triangular dither in (2) to be sufficiently small, it is possible to make the solution $x(t)$ of the relay feedback system arbitrarily close to the solution $w(t)$ of the smoothed system on any compact time interval.

Theorem III.1: Consider systems (1)-(3). Let T, $\varepsilon>0$ and $x_{0} \in \mathbb{R}^{n}$ be given. Assume that $r(t)$ is Lipschitz on $[0, T]$, with Lipschitz constant $M_{r}$. There exists $p_{0}>0$ such that if $p \in\left(0, p_{0}\right)$, then $\left|x\left(t, x_{0}\right)-w\left(t, x_{0}\right)\right| \leq \varepsilon$ for all $t \in[0, T]$.

Proof: See Appendix I.
Theorem III.1 can be interpreted as an extension of [3, Th. 1] to a class of nonsmooth systems. The result in [3] relies on continuity properties of the solutions of the original and the smoothed systems. This argument cannot be used here. Instead, we pay particular attention in the proof to the system evolution at and between relay switchings.

The proof of Theorem III. 1 is constructive, so a bound for $p_{0}$ is derived. It shows that $p_{0}$ should be chosen to be of the order of $\varepsilon$. The bound on $p_{0}$ depends on system data and $T$. It is conservative and tighter bounds will be obtained in Section III-D by exploiting more of the problem structure.

Theorem III. 1 holds also for the sawtooth dither [27] or skewtriangular dither.

\section{B. Practical Stability}

We use Theorem III.1 to obtain conditions for practical stability of the dithered system (1). The idea is the following. First choose the amplitude $A$ of the dither signal, such that the smoothed system in (3) is stable. Then, if the period $p$ of the dither signal is chosen to be small enough, the output of the dithered system closely follows the output of the smoothed system. This implies that the output of the dithered system converges close to zero. Note that we cannot obtain convergence strictly to zero, since the dither signal always cause small fluctuations of the output. We use the following definition of stability.

Definition III.1 (Practical Stability): The system (1) with triangular dither (2) and a given amplitude $A>0$ is practically (exponentially) stable, if for any $\varepsilon>0$, and $x_{0} \in \mathbb{R}^{n}$, there exist $\alpha>0, \beta \geq 1$, and $p_{0}>0$, such that

$$
|x(t)| \leq \beta e^{-\alpha t}\left|x_{0}\right|+\varepsilon \quad \forall t \in[0, \infty)
$$

for any dither period $p \in\left(0, p_{0}\right)$.

Theorem III.2: Suppose $r(t) \equiv 0$ and that the smoothed system (3) is exponentially stable. Then there exists $p_{0}$ such that for $p \in\left(0, p_{0}\right)$ the dithered system (1)-(2) is practically stable.

Proof: See Appendix II.

There are many available results for stability analysis of the smoothed system. We will here use a criterion by Zames and Falb [28], which generalizes the Popov criterion.

Corollary III.1: Assume that $L$ is Hurwitz. Let $G(j \omega)=$ $-c(j \omega I-L)^{-1} b$ and $H(j \omega)=\int_{-\infty}^{\infty} h(t) e^{-j \omega t} d t$, where $h$ : $\mathbb{R} \rightarrow \mathbb{R}$ satisfies $\int_{-\infty}^{\infty}|h(t)| d t \leq 1$. If there exists $\varepsilon>0$ such that

$$
\operatorname{Re}(G(j \omega)+A)(1+H(j \omega)) \geq \varepsilon \quad \forall \omega \in(0, \infty)
$$

then there exists $p_{0}$ such that for $p \in\left(0, p_{0}\right)$ the dithered system (1) is practically stable.

Proof: Inequality (5) gives a sufficient condition for the exponential stability of the smoothed system [28], [29]. By applying Theorem III.2, the corollary is then proven.

Note that the criterion (5) corresponds to one of the least conservative conditions for stability available for systems with a slope-restricted nonlinearity. However, it does not give any immediate information on the performance (e.g., the exponential decay parameters $\alpha$ and $\beta$ ), and it is not convex in the pair $(A, H)$. The circle criterion corresponds to $H=0$. From the Kalman-Yakubovich-Popov lemma, one can for that case derive an LMI that verifies (5) and results in explicit estimates of the 
exponential decay parameters. In Section IV, we will show how a suitable choice of $H$ can help to obtain better tuning of $A$.

\section{Infinite Time Horizon}

The next result shows that the dithered system can track the averaged system arbitrarily well over an infinite time horizon provided that the dither signal is chosen appropriately.

Let us call $x\left(t, r, x_{0}\right)$ the solution of (1)-(2) with reference $r$ and $w\left(t, r, w_{0}\right)$ the solution of (3).

Definition III.2: The smoothed system (3) is incrementally exponentially stable if there exists $\beta \geq 1$ and $\alpha>0$ such that for any given initial conditions $w_{1}$ and $w_{2}$, the corresponding solutions satisfy

$$
\left|w\left(t, r, w_{1}\right)-w\left(t, r, w_{2}\right)\right| \leq \beta e^{-\alpha t}\left|w_{1}-w_{2}\right| \quad \forall t \geq 0 .
$$

A simple and often very useful criterion for incremental exponential stability is given by the next lemma.

Lemma 1: Assume that there exists a matrix $Q>0$ and $\alpha>0$ such that the matrix inequality

$$
\left[\begin{array}{cc}
L^{T} Q+Q L+2 \alpha Q & Q b+c^{T} \\
b^{T} Q+c & -2 A
\end{array}\right] \leq 0
$$

holds. Then, the smoothed system (3) is incrementally exponentially stable with decay rate $\alpha$ and gain $\beta=\sqrt{\lambda_{\max }(Q) / \lambda_{\min }(Q)}$.

Theorem III.3: Consider systems (1)-(3). Let $\varepsilon>0$ and $x_{0} \in \mathbb{R}^{n}$ be given. Suppose that $r(t)$ has Lipschitz constant $M_{r}$ and the smoothed system (3) is incrementally exponentially stable. Then, there exists $p_{0}>0$ such that if $p \in\left(0, p_{0}\right)$, then $\left|x\left(t, x_{0}\right)-w\left(t, x_{0}\right)\right| \leq \varepsilon$ for all $t \in[0, \infty)$.

Proof: See Appendix III.

\section{Improved $p_{0}$ Bound}

We first improve the bound on $p_{0}$ obtained in the proof of Theorem III.1. A much tighter bound is then obtained when the smoothed system is incrementally stable.

Theorem III.4: Assume $r(t)$ has Lipschitz constant $M_{r}$. Suppose there exist $P=P^{T}>0$ and $\gamma>0$ such that

$$
\left[\begin{array}{ccc}
L^{T} P+P L & P b+c^{T} & 0 \\
b^{T} P+c & -2 A & c \\
0 & c^{T} & -\gamma I
\end{array}\right] \leq 0 .
$$

Then, the bound $p_{0}$ on the dither period in Theorem III.1 can be chosen to be

$$
p_{0}=\min \left(\frac{4 A}{7 M}, \Gamma^{-1} \varepsilon\right)
$$

where

$$
\begin{aligned}
\Gamma & =c_{3} T+2 c_{1}+\sqrt{\frac{\gamma}{\lambda_{\min }(P)}} \sqrt{\frac{1}{3 c_{3}}\left(\left(c_{3} T+2 c_{1}\right)^{3}-8 c_{1}^{3}\right)} \\
c_{1} & =\max _{t \in[0, T]}\left|e^{L t} b\right| \\
c_{2} & =\max _{t \in[0, T]}\left|L e^{L t} b\right| \\
c_{3} & =\frac{1}{2}\left(\frac{9 M}{A} c_{1}+3 c_{2}\right) \\
M & =\max _{t \in[0, T]}\left[\left|c L e^{L t} x_{0}\right|+\int_{0}^{t}\left|c L e^{L s} b\right| \mathrm{d} s+|c b|\right]+M_{r} .
\end{aligned}
$$

Proof: See Appendix IV.

By combining Theorems III.3 and Theorem III.4 we obtain much better bounds for the dither frequency, also in the case of infinite time horizon. We state this as the following theorem. Here, we assume that we have an estimate ${ }^{1} \Xi$ of the norm $\|w\|_{\infty}=\sup _{t \in[0, \infty)}|w(t)|$.

Theorem III.5: Let $\varepsilon>0$ and $x_{0} \in \mathbb{R}^{n}$ be given. Assume that $r(t)$ has Lipschitz constant $M_{r}$. Suppose that the smoothed system (3) is incrementally exponentially stable with decay rate $\alpha$ and gain $\beta$. Then, the bound $p_{0}$ on the dither period in Theorem III. 3 can be chosen to be

$$
p_{0}=\min \left(\frac{4 A}{7 M}, \frac{\Gamma^{-1} \varepsilon}{\left(1+\frac{\beta}{\mu}\right)}\right)
$$

where $\mu \in(0,1)$,

$$
\begin{aligned}
\Gamma= & \frac{c_{3}}{\alpha} \ln \left(\frac{\beta}{1-\mu}\right)+2 c_{1}+\sqrt{\frac{\gamma}{\lambda_{\min }(P)}} \\
& \cdot \sqrt{\frac{1}{3 c_{3}}\left(\left(\frac{c_{3}}{\alpha} \ln \left(\frac{\beta}{1-\mu}\right)+2 c_{1}\right)^{3}-8 c_{1}^{3}\right)}
\end{aligned}
$$

where $P>0$ solves the LMI in (8) and

$$
\begin{aligned}
& c_{1}=\max _{t \in\left[0, T^{*}\right]}\left|e^{L t} b\right| \\
& c_{2}=\max _{t \in\left[0, T^{*}\right]}\left|L e^{L t} b\right|, \\
& c_{3}=\frac{1}{2}\left(\frac{9 M}{A} c_{1}+3 c_{2}\right)
\end{aligned}
$$

$M=|c L|(\Xi+\varepsilon)+|c b|+M_{r}$, and $T^{*}=(1 / \alpha) \ln (\beta / 1-\mu)$.

Proof: The proof follows by using Theorem III.4 in an analogous reasoning as in Remark 1 in the proof of Theorem III.3.

Note that the parameter $M$ is a bound on the Lipschitz constant of $c x(t)+r(t)$. The bounds suggested in Theorems III.4 and III.5 can be conservative. The more knowledge we have about the trajectory of the smoothed system and the reference signal, the better bound we are able to obtain.

\section{DESIGN}

In this section, we use Theorems III.1 and III.2 to tune the dither signal. The purpose can, for example, be to stabilize an oscillating system. We use Theorem III.5 to obtain an LMIbased design methodology of the dither parameters. This results in an exponentially stable system with a state that tracks the state of the smoothed system with arbitrary precision. We finally present a heuristic method, which often gives less conservative designs. The design methods are illustrated in the example in Section II-C.

\section{A. First Tuning Algorithm}

The dither design will necessarily be a compromise between conflicting consequences of the dither amplitude $A$ and period

${ }^{1}$ Such a bound is easy to obtain for a given reference signal. If we have a class of reference signals, then we can obtain a bound by exploiting the incremental exponential stability of the smoothed system. 
$p$ on the control performance. Based on our results we obtain the following algorithm for tuning the previous parameters of the dither signal.

Step 1 Choose $A$ based on Corollary III.1, so that the smoothed system is exponentially stable.

Step 2 Estimate the exponential stability parameters $\alpha_{0}, \beta_{0}$ for the smoothed system by using the Kalman-Yakubovich-Popov Lemma. Let $T=-\ln \left(\alpha_{1} / \beta_{0}\right) / \alpha_{0}$, with $0<\alpha_{1}<1$. Step 3 Choose $p_{0}$ based on $T$ and the smoothed dynamics.

In Step 1, we need to choose the amplitude $A$ of the dither signal to be large enough to allow the smoothed system to be stable and to have a fast enough exponential decay rate. At the same time, we want to keep $A$ as small as possible in order to avoid injecting too large a signal in the control loop.

In Step 2, we compute the time-interval length $T$, which is an auxiliary variable in the proof of Theorem III. 2 and it depends on the parameters $\alpha_{0}, \beta_{0}$, and $\alpha_{1}$, a free parameter which represents a tradeoff between a low-ripple and a high-decay rate of the dithered system $\left(\alpha_{1} \rightarrow 0\right)$ and a low-dither frequency $\left(\alpha_{1} \rightarrow 1\right)$. The time length $T$ gives a bound on the period of the dither signal through (36) in the proof of Theorem III.1. Better bounds can be derived if we use the structure of the saturation nonlinearity and that the smoothed dynamics is chosen to be exponentially stable. The bound derived in Theorem III.4 is taking several of these structural aspects into account.

\section{B. A Second Tuning Algorithm}

We use Theorem III.5 to derive a tuning algorithm that gives an exponentially stable dither system, which tracks the state of the smoothed system over an infinite time horizon with any desired accuracy. We assume that we have derived a bound $\Xi$ of $\|w\|_{\infty}=\sup _{t \in[0, \infty)}\left|w\left(t, r, x_{0}\right)\right|$. For given tracking accuracy $\varepsilon$, Theorem III.5 gives $p_{0}$. We would like to optimize the free parameters such that $p_{0}$ becomes as small as possible. This is hard since the dependence on the free parameters in (9)-(10) is nonconvex. One way to obtain a solution is to pick $\mu \in(0,1)$ and $A$, and then choose a desired exponential decay rate such that the LMIs (7) and (8) are feasible. We see that $\beta$ and $\gamma / \lambda_{\min }(P)$ should be as small as possible to make $p_{0}$ small. This can be done by solving the following coupled optimization problems:

$$
\begin{aligned}
& \min \beta^{2} \\
& \text { s.t. } \\
& {\left[\begin{array}{cc}
L^{T} Q+Q L+2 \alpha Q & Q b+c^{T} \\
b^{T} Q+c & -2 A
\end{array}\right] \leq 0} \\
& \beta^{2} I \lambda_{\min } \geq Q \geq \lambda_{\min } I \\
& \min \bar{\lambda} \\
& \text { s.t. } \\
& \begin{array}{l}
{\left[\begin{array}{ccc}
L^{T} P+P L & P b+c^{T} & 0 \\
b^{T} P+c & -2 A & c \\
0 & c^{T} & -\gamma I
\end{array}\right] \leq 0} \\
\bar{\lambda} P \geq \gamma I>0 .
\end{array}
\end{aligned}
$$

The first problem can be solved by bisection on $\beta^{2}$ and the second by bisection on $\bar{\lambda}$. From the last optimization problem, we obtain $\gamma / \lambda_{\min }(P) \leq \bar{\lambda}$. Note that the constraints of these two optimization problems are LMIs for fixed $\beta^{2}$ and $\bar{\lambda}$, respectively. We have arrived at the following tuning algorithm.

Step 1) Choose $\mu \in(0,1)$.

Step 2) Choose a desired exponential decay rate $\alpha$ and then select the dither amplitude $A$ so that the LMIs (11b) and (12b) are feasible.

Step 3) Solve the optimization problem (11a), which gives $Q$ and $\beta$ and then problem (12a), which gives $P$ and $\gamma$.

Step 4) Compute $p_{0}$ from (9).

\section{A Heuristic Tuning Algorithm}

A practical issue that can be taken into account when tuning the dither period is how much fluctuation on the output we can allow due to the dither signal. We derive a heuristic bound on these fluctuations. Assume the transients have decayed so that we can consider the linear range of the smoothed nonlinearity. Then, the transfer function

$$
G_{\mathrm{cl}}(s)=\left(1+\frac{G(s)}{A}\right)^{-1} \frac{G(s)}{A}
$$

where $G(s)=-c(s I-L)^{-1} b$ approximately describes the mapping from the dither signal to the output $y=-c x$. Choose $\omega_{0}>0$ such that

$$
\left|G_{\mathrm{cl}}(j \omega)\right| \leq \frac{\eta}{A} \quad \forall \omega \geq \omega_{0}
$$

for some small $\eta>0$. Then, we can expect $|y(t)| \leq \eta$ for sufficiently large $t$, if the dither period is chosen such that $p_{0} \leq$ $2 \pi / \omega_{0}$. The following heuristic tuning rule follows.

Step 1) Choose an output bound $\eta>0$.

Step 2) Choose $A$ based on Theorem III.2.

Step 3) Choose $p_{0} \leq 2 \pi / \omega_{0}$, where $\omega_{0}$ satisfies (13).

We have assumed the dither signal to be approximately sinusoidal while deriving this bound. Analytical expressions for the stationary periodic oscillation in a dithered relay system can be derived using the same approach as for relay feedback systems with no dither [20]-[22], [24], [25]. This gives the exact dither ripple, see [27].

\section{Example Revisited}

Let us continue discussing the example in Section II-C. Recall that

$$
G(s)=-c(s I-L)^{-1} b=\frac{1-s}{(s+1)^{2}} .
$$

In all the tuning algorithms, the first step is to choose the dither amplitude $A$. Consider Theorem III.2 with $H(s)=0$, which corresponds to the circle criterion. For $A>0.56$, we have $\operatorname{Re} G(j \omega)+A>0 \forall \omega \in[0, \infty)$. Hence, the dithered system is practically stable for $A>0.56$ and $p$ sufficiently small. By using Theorem III. 2 with $H(s)=-(s+1)^{-1}$ instead, we can prove practical stability for $A>0.501$. The first two tuning algorithms with $A=1$ give $p_{0} \simeq 1 / 10^{8}$ and $p_{0} \simeq 1 / 10^{4}$, respectively, which are both quite conservative bounds. The heuristic tuning algorithm gives the better estimate $p_{0} \simeq 1 / 3$. 

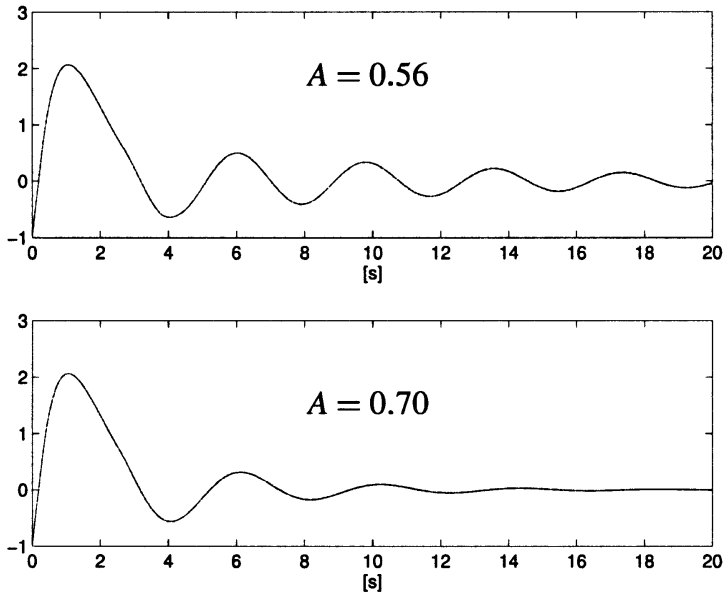

Fig. 2. Output of the dithered system with $\delta$ having period $p=1 / 50$. The amplitude is $A=0.56$ (upper) and $A=0.70$ (lower), respectively. A smaller $A$ thus gives a less oscillating response.

The effect of the dither period $p$ on the responses of the dithered and smoothed systems are shown in Fig. 1. The effects of the dither amplitude $A$ are shown in Fig. 2. It is possible to obtain a fast convergence by increasing $A$.

\section{COUNTER EXAMPLES FOR OTHER Dither Signals}

The averaging theorems in Section III cannot be directly extended to dither signals that have zero slope in a time interval of nonzero measure. Indeed, a closer look at the proof of Theorem III.1 shows that the approximation error is larger than of order $p$. One would therefore conjecture that averaging may not apply to such dither signals. We next give two examples that support this conjecture.

In the first example, we consider system (1) with squarewave dither of period $p$ and amplitude $A$, and a constant external reference $r=R$. In this case, the smoothed nonlinearity is a dead-band relay. Fig. 3(a) shows the output of the relay feedback system dithered with a squarewave and the output of the corresponding smoothed system. The waveforms do not change when the dither period is decreased. There remains a limit cycle in the dithered system for all periods $p$ we investigated. This example indicates that the error between the dithered and the smoothed system is not of order $p$, which would be the case if we use a triangular dither signal.

In our second example, we consider a trapezoidal dither signal with slope equal to 1000. It can be shown that the corresponding smoothed nonlinearity is discontinuous in $z=-A$ and $z=+A$ but is linear in the region $|z|<A$ [27], [30], [31]. The smoothed and the dithered systems have output waveforms that are highly different in time, see Fig. 3(b). We can see that the stationary behaviors of the systems are periodic but the period of the smoothed system is different from the period of the dithered system.

These simulation studies show that averaging may not take place when we use dither signals that have zero slope on intervals of nonzero measure. Indeed, the examples show that the behavior of the dithered and the smoothed systems can be very

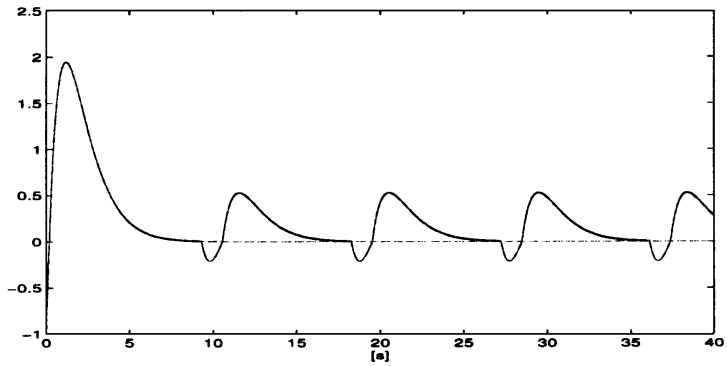

(a)

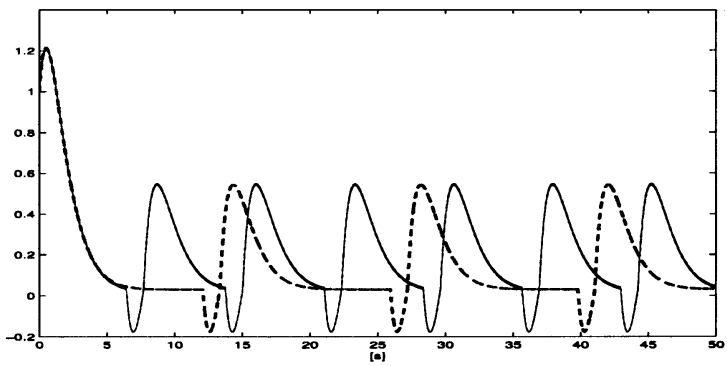

(b)

Fig. 3. Outputs of the dithered relay feedback system (solid) and the smoothed system (dashed) with square and trapezoidal dither signals ( $p=1 / 50, A=1$ ). (a) Square dither and $r(t)=1$. (b) Trapezoidal dither and $r(t)=1.02$.

different when such dither signals are used. A detailed analysis of such behaviors is out of the scope of this paper. We have done some preliminary work in this direction [27], [30] and we have verified that these type of phenomena also can appear in laboratory experiments [31]. However, further investigation on the behavior of relay feedback systems with various dither signals is needed.

\section{CONCLUSION}

In this paper, we have shown how dither can be analyzed in nonsmooth systems. The main approach is that a relay feedback system with a triangular dither signal at the input of the relay can be viewed as a feedback system without dither in which the relay is replaced by a saturation. The amplitude of the dither signal affects the slope of the saturation. The approximation of the dithered system by the smoothed system depends on the frequency of the dither signal. Explicit algorithms to achieve a desired approximation error have been given. Furthermore, analytical and practical guidelines to design dithered systems have been presented. These were verified by simulations. Finally, it has been shown that for the class of dither signals with zero slope in nonzero time intervals the averaging might not work as for dither signals like triangular, sawtooth, or skew triangular.

\section{APPENDIX I PROOF OF THEOREM III.1}

Consider the dithered system (1) and the smoothed system (3) on the time interval $[0, T]$ and with $w(0)=x(0)=x_{0}$

$$
\begin{aligned}
& \dot{x}(t)=L x(t)+b n(c x(t)+r(t)+\delta(t)), \quad x(0)=x_{0} \\
& \dot{w}(t)=L w(t)+b N(c w(t)+r(t)), \quad w(0)=x_{0} .
\end{aligned}
$$


Note that the right-hand side of (14a) is bounded on every compact time interval $[0, T]$, so that there exists a positive constant $M_{y}$ such that $|c \dot{x}(t)| \leq M_{y}$, for almost every $t \in[0, T]$

$M_{y}\left(x_{0}\right):=\max _{t \in[0, T]}\left[\left|c L e^{L t}\right| \cdot\left|x_{0}\right|+\int_{0}^{t}\left|c L e^{L(t-s)} b\right| \mathrm{d} s+|c b|\right]$.

Moreover, by hypothesis $r(t)$ is Lipschitz

$$
\left|r\left(t_{1}\right)-r\left(t_{2}\right)\right| \leq M_{r}\left|t_{1}-t_{2}\right| \quad \forall t_{1}, t_{2} .
$$

Then, we introduce $M=M_{y}+M_{r}$. By integrating the two members of (14), we obtain

$$
\begin{aligned}
x(t)-w(t)= & L \int_{0}^{t}[x(s)-w(s)] \mathrm{d} s \\
& +b \int_{0}^{t}[n(c x(s)+r(s)+\delta(s)) \\
& -N(c w(s)+r(s))] \mathrm{d} s .
\end{aligned}
$$

The idea now is to show that the integral $\int_{0}^{t}[n(c x(s)+r(s)+$ $\delta(s))] \mathrm{d} s$ can be approximated by $\int_{0}^{t} N(c x(s)+r(s)) \mathrm{d} s$. The error introduced by this approximation is a function of the dither period $p$. We will show that it can be made small by decreasing the period $p$.

We first evaluate the term $\int_{0}^{t}[n(c x(s)+r(s)+\delta(s))] \mathrm{d} s$. If we introduce $m=\lfloor T / p\rfloor$, the largest integer such that $m p \leq T$, then

$$
\begin{aligned}
\int_{0}^{t} n(c x(s) & +r(s)+\delta(s)) \mathrm{d} s \\
= & \int_{m p}^{m p+\Delta t} n(c x(s)+r(s)+\delta(s)) \mathrm{d} s \\
& +\sum_{k=0}^{m-1} \int_{k p}^{(k+1) p} n(c x(s)+r(s)+\delta(s)) \mathrm{d} s
\end{aligned}
$$

with $\Delta t=T-m p$. Since $n$ is a bounded function and the time interval of the last integral in (17) has a Lebesgue measure less than $p$, we can write

$$
\begin{aligned}
\int_{0}^{t} n & (c x(s)+r(s)+\delta(s)) \mathrm{d} s \\
& =\sum_{k=0}^{m-1} \int_{k p}^{(k+1) p} n(c x(s)+r(s)+\delta(s)) \mathrm{d} s+V_{0}(t)
\end{aligned}
$$

with $\left|V_{0}(t)\right| \leq p$. Each term in the sum can be written as

$$
\begin{aligned}
\int_{0}^{p} n(c x(s+k p) & +r(s+k p)+\delta(s+k p)) \mathrm{d} s \\
= & p N(c x(k p)+r(k p)) \\
& +\int_{0}^{p}[n(c x(s+k p)+r(s+k p)+\delta(s)) \mathrm{d} s \\
& -\int_{0}^{p} n(c x(k p)+r(k p)+\delta(s)) \mathrm{d} s
\end{aligned}
$$

Fig. 4 illustrates the evolution for one dither period interval. In the top diagram, the solid lines bound $c x(s+k p)+r(s+k p)+$ $\delta(s), 0 \leq s \leq p$. The dashed line is $c x(k p)+r(k p)+\delta(s)$. The figure presents all possible cases for the evolution of $c x+r+\delta$, in the sense that the envelope has the same characteristics as

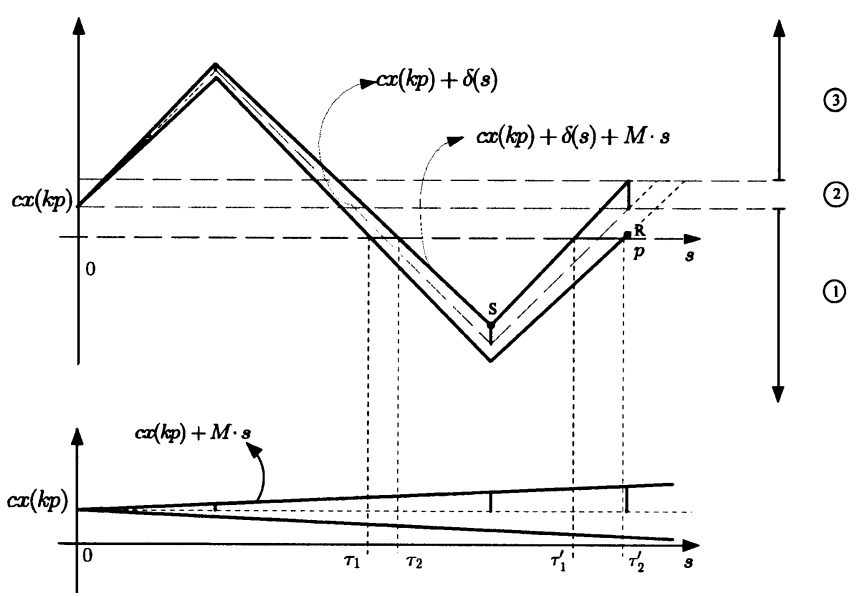

Fig. 4. Time diagrams of the signals.

long as the point $\mathbf{R}$ is above the point $\mathbf{S}$. It is not difficult to show that this is equivalent to

$$
p<\frac{1}{7} \cdot \frac{4 A}{M}=: \bar{p} .
$$

In the following, we assume that $p$ is chosen such that (20) holds.

All possible cases correspond to different values of $c x(k p)+$ $r(k p)$ or, equivalently, all possible cases can be obtained by shifting the horizontal $s$ axis upward and downward in the top diagram of Fig. 4. We have three cases

$$
\begin{cases}0 \leq c x(k p)+r(k p) & \text { Region 1 } \\ c x(k p)+r(k p) \leq 0 \leq c x(k p)+r(k p)+M p & \text { Region 2 } \\ c x(k p)+r(k p)+M p \leq 0 & \text { Region 3. }\end{cases}
$$

The regions are illustrated to the right in Fig. 4 by the location of the $s$ axis for the three cases. The partition identifies the time intervals, during which the signal $c x(s+k p)+r(s+k p)+$ $\delta(s)$ can have a zero crossing. It is only during these intervals that the sum of two integrals in (19) can be different from zero. Introduce $I_{i}$ to denote the sum of the lengths of these intervals for Region $i$, as further described below. Next, we discuss each region separately.

Region 1: For the first region, $I_{1}$ is equal to

$$
I_{1}=\frac{3}{2} \cdot \frac{M}{2 A} \cdot \frac{p^{2}}{1-\left(\frac{M p}{(4 A)}\right)^{2}} .
$$

Region 3: In this case

$$
I_{3}=\frac{1}{2} \cdot \frac{M}{2 A} \cdot \frac{p^{2}}{1-\left(\frac{M p}{(4 A)}\right)^{2}} .
$$

Note that both $I_{1}$ and $I_{3}$ are independent from the value of $c x(k p)+r(k p)$.

Region 2: Finally, we consider the second region. It is possible to derive the following bound:

$$
I_{2} \leq I_{1}+I_{3}=2 \cdot \frac{M}{2 A} \cdot \frac{p^{2}}{1-\left(\frac{M p}{(4 A)}\right)^{2}} .
$$

To conclude the discussion on Regions $1-3$, note that the worst case $I$, say, for all three of them is bounded by the right-hand side of (23). It is easy to see that there exists $p^{*}>0$ such that for all $p \leq p^{*}$, we have $I$ of ordo $p^{2}$, i.e., $I=O\left(p^{2}\right)$. 
In particular, we may choose

$$
p^{*}=\frac{4 A}{M} \cdot \frac{\sqrt{2}}{2}
$$

so that

$$
I \leq 4 \cdot \frac{M}{2 A} p^{2} \quad \forall p \leq p^{*} .
$$

Note that (25) follows from (20). In conclusion, the estimate of the upperbound (25) is valid for all cases, hence we have that (19) is equal to

$$
\begin{aligned}
\int_{k p}^{(k+1) p} n(c x(s) & +r(s)+\delta(s)) \mathrm{d} s \\
= & p N(c x(k p)+r(k p))+Z_{1}(k), p \leq \bar{p}
\end{aligned}
$$

with $\left|Z_{1}(k)\right| \leq 8 \cdot(M / 2 A) p^{2}$.

So far, we have mainly considered one period $p$. Since in (18), we have $m=\lfloor T / p\rfloor$ terms, we have

$$
\begin{aligned}
\int_{0}^{t} n(c x(s)+r(s)+\delta(s)) \mathrm{d} s= & \sum_{k=0}^{m-1} p N(c x(k p)+r(k p)) \\
& +V_{0}(t)+V_{1}(t)
\end{aligned}
$$

with $\left|V_{1}(t)\right| \leq 8 \cdot(M / 2 A) T p$. For a sufficiently small value of $p$, (or equivalently, for a sufficiently large value of $m$ ), the sum can be approximated by an integral. The maximum error of the approximation is related to the maximum slope of the signal $N(c x(s)+r(s))$. However, $N$ satisfies the slope condition

$$
0 \leq\left|N\left(c x\left(s_{1}\right)+r\left(s_{1}\right)\right)-N\left(c x\left(s_{2}\right)+r\left(s_{2}\right)\right)\right| \leq \frac{M}{A}\left|s_{1}-s_{2}\right|
$$

for all $s_{1}, s_{2} \in[0, T]$, which implies

$$
\int_{k p}^{(k+1) p} N(c x(s)+r(s)) \mathrm{d} s=p N(c x(k p)+r(k p))+Z_{2}(k)
$$

(with $\left|Z_{2}(k)\right| \leq(M / 2 A) p^{2}$ ) and, thus

$$
\begin{aligned}
\sum_{k=0}^{m-1} p N(c x(k p)+r(k p))= & \int_{0}^{m p} N(c x(s)+r(s)) \mathrm{d} s+V_{2}(t) \\
= & \int_{0}^{t} N(c x(s)+r(s)) \mathrm{d} s \\
& +V_{2}(t)+V_{3}(t)
\end{aligned}
$$

with $\left|V_{2}(t)\right| \leq(M / 2 A) T p$ and $\left|V_{3}(t)\right| \leq p$.

We have up to now proved that (16) can be written as

$$
\begin{aligned}
x(t)-w(t)= & L \int_{0}^{t}[x(s)-w(s)] \mathrm{d} s \\
& +b \int_{0}^{t}[N(c x(s)+r(s)) \\
& -N(c w(s)+r(s))] \mathrm{d} s+V(t)
\end{aligned}
$$

for all $p \leq \bar{p}$ where $V(t)=b\left(V_{0}(t)+V_{1}(t)+V_{2}(t)+V_{3}(t)\right)$ and

$$
\begin{aligned}
|V(t)| & \leq|b|\left(p+8 \cdot \frac{M}{2 A} T p+\frac{M}{2 A} T p+p\right) \\
& =|b|\left(\frac{9}{2} \frac{M}{A} T+2\right) p .
\end{aligned}
$$

Since $N$ has the Lipschitz constant equal to $1 / A$, we get

$|x(t)-w(t)| \leq\left(\|L\|+\frac{|b| \cdot|c|}{A}\right) \int_{0}^{t}|x(s)-w(s)| \mathrm{d} s+O(p)$

for $p \leq \bar{p}$ where

$$
O(p)=\left(\frac{9}{2} \cdot \frac{M}{A} T+2\right)|b| p .
$$

Now, by applying the Grönvall-Bellman lemma [32] to (32), we get for all $p \leq \bar{p}$

$$
\begin{aligned}
|x(t)-w(t)| \leq & O(p)+O(p)\left(\|L\|+\frac{|b| \cdot|c|}{A}\right) \\
& \cdot \int_{0}^{t} e^{(\|L\|+(|b| \cdot|c| / A))(t-\tau)} d \tau \\
= & O(p)+O(p)\left[e^{(\|L\|+(|b| \cdot|c| / A)) t}-1\right] .
\end{aligned}
$$

Hence

$$
\begin{aligned}
|x(t)-w(t)| & \leq O(p) e^{(\|L\|+|b| \cdot|c| / A) t} \\
& \leq O(p) e^{(\|L\|+|b| \cdot|c| / A) T} \\
& =\varepsilon \quad \forall t \in[0, T] .
\end{aligned}
$$

This concludes the proof of the theorem.

Note that from (33), we have an estimate of $p_{0}$ of the theorem, namely

$$
p_{0}=\min \left(\frac{4 A}{7 M}, \frac{\varepsilon}{\left(\frac{9 M T}{(2 A)}+2\right)|b| e^{(\|L\|+|b| \cdot|c| / A) T}}\right) .
$$

\section{APPENDIX II \\ PROOF OF THEOREM III.2}

By hypothesis, (3), with $r(t) \equiv 0$, is exponentially stable. Hence, there exists $\alpha_{0}>0$ and $\beta_{0} \geq 1$ such that

$$
|w(t)| \leq \beta_{0} e^{-\alpha_{0} t}\left|x_{0}\right| \quad \forall t \geq 0 .
$$

We will use this to prove the practical stability of (1). We iteratively consider time intervals of length $T=-\alpha_{0}{ }^{-1} \ln \left(\alpha_{1} / \beta_{0}\right)$ where $0<\alpha_{1}<1$. Then, if $p_{0}$ is sufficiently small (see (36)), we have

$$
|x(t)-w(t)| \leq \varepsilon_{0}
$$

on $t \in[0, T]$. If we consider a sequence of variables $\tilde{w}_{k}, k=$ $1,2, \ldots$, each defined on an interval $[k T,(k+1) T]$ and satisfying (3) with $\tilde{w}_{k}(k T)=x(k T)$, then it follows that

$$
\left|\tilde{w}_{k}(t)\right| \leq \beta_{0} e^{-\alpha_{0}(t-k T)}|x(k T)| \quad \forall t \geq k T .
$$

By applying Theorem III.1 again

$$
|x(t)|=\left|x(t)-\tilde{w}_{k}(t)+\tilde{w}_{k}(t)\right| \leq \varepsilon_{0}+\beta_{0} e^{-\alpha_{0}(t-k T)}|x(k T)|
$$

on $t \in[k T,(k+1) T]$. By evaluating (37) in $t=(k+1) T$

$$
|x((k+1) T)| \leq \alpha_{1}|x(k T)|+\varepsilon_{0} .
$$

Hence

$$
|x(k T)| \leq \alpha_{1}^{k}\left|x_{0}\right|+\varepsilon_{0} \frac{1-\alpha_{1}^{k}}{1-\alpha_{1}} .
$$


Then, (37) becomes

$$
\begin{aligned}
|x(t)| & \leq \beta_{0} e^{-\alpha_{0}(t-k T)}\left(e^{-\alpha k T}\left|x_{0}\right|+\frac{\varepsilon_{0}}{1-\alpha_{1}}\right)+\varepsilon_{0} \\
& \leq \beta_{0} e^{-\alpha t}\left|x_{0}\right|+\beta_{0} \frac{\varepsilon_{0}}{1-\alpha_{1}}+\varepsilon_{0}
\end{aligned}
$$

where $\alpha=-T^{-1} \ln \alpha_{1}<\alpha_{0}$. We have thus shown practical stability with $\alpha=-T^{-1} \ln \alpha_{1}$ and $\beta=\beta_{0}$.

\section{APPENDIX III \\ PROOF OF THEOREM III.3}

We will need to consider the dithered and the smoothed system from arbitrary initial time $t_{0} \geq 0$. Hence, for the proof we consider the dithered system

$$
\left\{\begin{array}{l}
\dot{x}(t)=L x(t)+b n(c x(t)+\delta(t)+r(t)) \quad \forall t \geq t_{0} \\
x\left(t_{0}\right)=x_{0}
\end{array}\right.
$$

and the smoothed system

$$
\left\{\begin{aligned}
\dot{w}(t) & =L w(t)+b N(c w(t)+r(t)) \quad \forall t \geq t_{0} \\
w\left(t_{0}\right) & =w_{0}
\end{aligned}\right.
$$

where $N(z)=\operatorname{sat}(z / A)$. We suppress the variable $r(t)$ and denote $x\left(t, t_{0}, x_{0}\right)$ the solution of (41) with initial condition $x\left(t_{0}\right)=x_{0}$ and $w\left(t, t_{0}, w_{0}\right)$ the solution of (42) with initial condition $w\left(t_{0}\right)=w_{0}$. We want to evaluate the approximation error $\left|x\left(t, t_{0}, x_{0}\right)-w\left(t, t_{0}, x_{0}\right)\right| \forall t \geq t_{0}$. For this, we will make critical use of the incremental exponential stability assumption which means that

$$
\left|w\left(t, t_{0}, w_{1}\right)-w\left(t, t_{0}, w_{2}\right)\right| \leq \beta e^{-\alpha\left(t-t_{0}\right)}\left|w_{1}-w_{2}\right| .
$$

Let us indicate as $w^{0}(t)$, the solution $w\left(t, t_{0}, x_{0}\right)$ of the problem (42). In the following, we will denote the time instant $k T$ as $T_{k}$ and the time instant $(k+1) T$ as $T_{k+1}$. The idea is to show that for each time interval $\left[T_{k}, T_{k+1}\right], k \geq 0, T>0$ the approximation error $\left|x\left(t, t_{0}, x_{0}\right)-w^{0}(t)\right| \quad t \in\left[T_{k}, T_{k+1}\right]$ is bounded by a function of order $p_{0}$.

Let us call $\tilde{w}(t, \bar{t})=w(t, \bar{t}, x(\bar{t})) t \geq \bar{t}$ the solution of the smoothed system (42) when the initial condition (at the time instant $t_{0}=\bar{t}$ ) is equal to the value that the state of dithered system assumes at $t=\bar{t}$. In other words, during each time interval $\left[T_{k}, T_{k+1}\right], \tilde{w}(t)$ is the solution of the smoothed system when the initial condition is equal to $x\left(T_{k}\right)$.

By the triangle inequality we have

$$
\left|x\left(t, 0, x_{0}\right)-w^{0}(t)\right| \leq\left|x\left(t, 0, x_{0}\right)-\tilde{w}(t)\right|+\left|\tilde{w}(t)-w\left(t, 0, x_{0}\right)\right|
$$

for all $t \in[0,+\infty)$. Let us consider the first time interval [0, $T$ ]. In this case, $\tilde{w}(t)=w^{0}(t)$ since the initial condition is the same for both the solutions. Hence, (44) reduces to

$$
\left|x\left(t, 0, x_{0}\right)-w^{0}(t)\right|=\left|x\left(t, 0, x_{0}\right)-\tilde{w}(t)\right| .
$$

We can apply Theorem III. 1 and choose a $p_{0}=p_{0}\left(x_{0}, T, M_{r}, \mu \xi\right)$ (where $x_{0}, T, M_{r}$, and $\varepsilon=\mu \xi$ are the parameters that define $p_{0}$ in (36) since $M=M_{y}\left(x_{0}\right)+M_{r}$ and $M_{y}\left(x_{0}\right)$ is defined in (15)) such that the approximation error is bounded by the value $\mu \xi$ with $0<\mu<1$ and $\xi>0$

$$
\left|x\left(t, 0, x_{0}\right)-w^{0}(t)\right|=\left|x\left(t, 0, x_{0}\right)-\tilde{w}(t)\right| \leq \mu \xi<\xi \forall t \in[0, T] .
$$

In particular, $\left|x\left(T, 0, x_{0}\right)-w^{0}(T)\right| \leq \xi$.
Now, let us consider a generic time interval $\left[T_{k}, T_{k+1}\right]$ with $\left|x\left(T_{k}, 0, x_{0}\right)-w^{0}\left(T_{k}\right)\right| \leq \xi$ (note that this inequality holds for $k=1)$

$$
\begin{aligned}
& \left|x\left(t, 0, x_{0}\right)-w^{0}(t)\right| \\
& =\left|x\left(t, T_{k}, x\left(T_{k}\right)\right)-w\left(t, T_{k}, w\left(T_{k}\right)\right)\right| \\
& \leq\left|x\left(t, T_{k}, x\left(T_{k}\right)\right)-\tilde{w}\left(t, T_{k}\right)\right|+\left|\tilde{w}\left(t, T_{k}\right)-w\left(t, T_{k}, w\left(T_{k}\right)\right)\right|
\end{aligned}
$$

for all $t \in\left[T_{k}, T_{k+1}\right]$. For the first term, we apply Theorem III. 1 on the finite time horizon on the interval $\left[T_{k}, T_{k+1}\right]$. It shows that there exists $p_{0}\left(x\left(T_{k}\right), T, M_{r}, \mu \xi\right)$, which gives the upper bound $\mu \xi$. For the second term, we use the incremental exponential stability condition in (43), which implies

$$
\begin{aligned}
\left|\tilde{w}\left(t, T_{k}\right)-w\left(t, T_{k}, w\left(T_{k}\right)\right)\right| & \leq \beta e^{-\alpha\left(t-T_{k}\right)}\left|x\left(T_{k}\right)-w\left(T_{k}\right)\right| \\
& \leq \beta e^{-\alpha\left(t-T_{k}\right)} \xi \leq \beta \xi
\end{aligned}
$$

for all $t \in\left[T_{k}, T_{k+1}\right]$. In the time instant $t=T_{k+1}$, we have

$$
\begin{aligned}
\left|x\left(T_{k+1}, 0, x_{0}\right)-w^{0}\left(T_{k+1}\right)\right| & \\
\leq & \left|x\left(T_{k+1}, T_{k}, x\left(T_{k}\right)\right)-\tilde{w}\left(T_{k+1}, T_{k}\right)\right| \\
& \quad+\left|\tilde{w}\left(T_{k+1}, T_{k}\right)-w\left(T_{k+1}, T_{k}, w\left(T_{k}\right)\right)\right| \\
\leq & \mu \xi+\beta e^{-\alpha T} \xi .
\end{aligned}
$$

If we choose a $T \geq(1 / \alpha) \ln (\beta /(1-\mu))$, then, the inequality (46) can be written as

$$
\left|x\left(T_{k+1}, 0, x_{0}\right)-w^{0}\left(T_{k+1}\right)\right| \leq \mu \xi+(1-\mu) \xi=\xi .
$$

We have shown that there exists $T>0$ and $p_{0}\left(x\left(T_{k}\right), T, M_{r}, \mu \xi\right)$ such that if $\left|x\left(T_{k}, 0, x_{0}\right)-w^{0}\left(T_{k}\right)\right| \leq \xi$, then, the approximation error is bounded from above by the value $\xi(\mu+\beta)$ on $\left[T_{k}, T_{k+1}\right]$ and $\left|x\left(T_{k+1}, 0, x_{0}\right)-w^{0}\left(T_{k+1}\right)\right| \leq \xi$. The infinite horizon theorem now follows by continuing this process inductively. Indeed, the incremental exponential stability assumption can be used to show that $\|w\|_{\infty}=\sup _{t \in[0, \infty)}\left|w\left(t, 0, x_{0}\right)\right|<\infty$ and a corresponding bound $\Xi$ of $\left\|w\left(t, 0, x_{0}\right)\right\|_{\infty}$ can be derived. Hence, we can use the bound $p_{0}=p_{0}\left(\Xi+\xi, T, M_{r}, \varepsilon\right)$ in each inductive step.

Remark 1: If we introduce $\kappa=(|| L \|+(|b| \cdot|c| / A))$ and use $T=(1 / \alpha) \ln (\beta / 1-\mu)$, by using (33) and (35), we obtain the approximation error

$$
\begin{aligned}
\varepsilon & =(\mu+\beta) \xi \\
& =\left(1+\frac{\beta}{\mu}\right)\left(4.5 \frac{M}{A} \frac{1}{\alpha} \ln \frac{\beta}{1-\mu}+2\right)|b|\left(\frac{\beta}{1-\mu}\right)^{\kappa / \alpha} p_{0}
\end{aligned}
$$

where $M=M_{y}(\Xi+\mu \xi)+M_{r}$, where $M_{y}(\cdot)$ is defined in (15). The approximation error is independent from the length of the time interval, and depends only on the free parameter $\mu$ that can be chosen such that the expression (48) is minimized.

\section{APPENDIX IV \\ PROOF OF THEOREM III.4}

The differential form of (31) is

$$
\begin{aligned}
\dot{x}(t)-\dot{w}(t)= & L(x(t)-w(t))+b[N(c x(t)+r(t)) \\
& -N(c w(t)+r(t))]+b v(t)
\end{aligned}
$$

where $v(t)=n(c x(t)+r(t)+\delta(t))-N(c x(t)+r(t))$. This 
system can equivalently be written

$$
\begin{aligned}
x(t)-w(t)= & \int_{0}^{t} e^{L(t-s)} b(N(c x(s)+r(s))-N(c w(s) \\
& +r(s))) \mathrm{d} s+e(t)
\end{aligned}
$$

where $e(t)=\int_{0}^{t} e^{L(t-s)} b v(s) \mathrm{d} s$.

Lemma 2: We have

$$
|e(t)| \leq\left(\left(\frac{9 M t}{2 A}+2\right) c_{1}+\frac{3 t}{2} c_{2}\right) p=\left(c_{3} t+2 c_{1}\right) p
$$

for all $t \in[0, T]$. Moreover,

$$
\int_{0}^{t}|e(s)|^{2} \mathrm{~d} s \leq \frac{1}{3 c_{3}}\left(\left(c_{3} t+2 c_{1}\right)^{3}-8 c_{1}^{3}\right) p^{2}
$$

where

$$
\begin{aligned}
c_{1} & =\max _{t \in[0, T]}\left|e^{L t} b\right| \\
c_{2} & =\max _{t \in[0, T]}\left|L e^{L t} b\right| \\
c_{3} & =\frac{1}{2}\left(\frac{9 M}{A} c_{1}+3 c_{2}\right) .
\end{aligned}
$$
and

$e_{1}(t)=\int_{m p}^{t} e^{L(t-s)} b[n(c x(s)+r(s)+\delta(s))-N(c x(s)+r(s))] \mathrm{d} s$

$e_{2}(t)=\sum_{k=0}^{m-1} \int_{k p}^{(k+1) p}\left[e^{L(t-s)} b n(c x(s)+r(s)+\delta(s))\right] \mathrm{d} s$

$-\sum_{k=0}^{m-1} \int_{k p}^{(k+1) p}\left[e^{L(t-k p)} b n(c x(k p)+r(k p)+\delta(s))\right] \mathrm{d} s$

$e_{3}(t)=\sum_{k=0}^{m-1} e^{L(t-k p)} b N(c x(k p)+r(k p)) p$

$-\int_{0}^{m p} e^{L(t-s)} b N(c x(s)+r(s)) \mathrm{d} s$.

For the first term, we have $\left|e_{1}(t)\right| \leq 2 c_{1} p$. Each term of $e_{2}$ can be split into two terms $e_{2}=\sum_{k=0}^{m-1}\left(e_{2_{k a}}+e_{2_{k b}}\right)$

$e_{2_{k a}}=\int_{k p}^{(k+1) p}\left(e^{L(t-s)}-e^{L(t-k p)}\right) b n(c x(s)+r(s)+\delta(s)) \mathrm{d} s$

$e_{2_{k b}}=\int_{k p}^{(k+1) p} e^{L(t-k p)} b[n(c x(s)+r(s)+\delta(s))] \mathrm{d} s$

$-\int_{k p}^{(k+1) p} e^{L(t-k p)} b[n(c x(k p)+r(k p)+\delta(s))] \mathrm{d} s$.

For the first, we get the bound $\left|e_{2_{k a}}\right| \leq c_{2} p^{2}$ and for the second, we get

$$
\left|e_{2_{k b}}\right| \leq c_{1} 4 \frac{M}{A} p^{2}, \quad p \leq \bar{p}
$$

which follows from (26) in the proof of Theorem III.1. If we put these bounds together and sum, we get

$$
\left|e_{2}(t)\right| \leq\left(c_{2}+c_{1} 4 \frac{M}{A}\right) t p
$$

for all $t \in[0, T]$. Finally, since the Lipschitz constant of $H(s)=$ $e^{L(t-s)} b N(c x(s)+r(s))$ can be bounded as

$$
\operatorname{Lip}[H] \leq c_{2}+c_{1} \frac{M}{A}
$$

and a similar argument as in (28)-(30) gives

$$
\left|e_{3}(t)\right| \leq\left(c_{2}+c_{1} \frac{M}{A}\right) \frac{t}{2} p \quad \forall t \in[0, T] .
$$

If we put everything together, we get

$$
|e(t)| \leq\left(\left(\frac{9 M t}{2 A}+2\right) c_{1}+\frac{3 t}{2} c_{2}\right) p \quad \forall t \in[0, T] .
$$

A state-space realization of (49) is

$$
\begin{aligned}
\dot{z}(t) & =L z(t)+b u(t) \\
z(0) & =x(0)-w(0)=0 \\
u(t) & =N(c x(t)+r(t))-N(c w(t)+r(t)) \\
x(t)-w(t) & =z(t)+e(t) .
\end{aligned}
$$

We will use the slope condition on the saturation nonlinearity $N$, which gives the relation

$$
(c(z+e)-A u) u \geq 0 .
$$

Now, let us multiply the LMI with $\left[\begin{array}{lll}z^{T} & u & e^{T}\end{array}\right]$ on the left, and by its transpose on the right. This gives

$$
\frac{d}{d t} z^{T} P z+2(c(z+e)-A u) u-\gamma e^{T} e \leq 0 .
$$

If we integrate this inequality and use that the second term is positive due to (50) then we get

$$
z(t)^{T} P z(t) \leq z(0)^{T} P z(0)+\gamma \int_{0}^{t}|e(s)|^{2} \mathrm{~d} s .
$$

Hence, using $z(0)=0$

$$
\begin{aligned}
|z(t)| & \leq \sqrt{\frac{\lambda_{\max }(P)}{\lambda_{\min }(P)}}|z(0)|+\sqrt{\frac{\gamma}{\lambda_{\min }(P)}} \sqrt{\int_{0}^{t}|e(s)|^{2} \mathrm{~d} s} \\
& \leq \sqrt{\frac{\gamma}{\lambda_{\min }(P)}} \sqrt{\frac{1}{3 c_{3}}\left(\left(c_{3} T+2 c_{1}\right)^{3}-8 c_{1}^{3}\right) \cdot p .}
\end{aligned}
$$

We see that $|x(t)-w(t)| \leq \varepsilon$ on $t \in[0, T]$ if $|z(t)|+|e(t)| \leq \varepsilon$, which is the case if $p \leq \Gamma^{-1} \varepsilon$ where

$$
\Gamma=c_{3} T+2 c_{1}+\sqrt{\frac{\gamma}{\lambda_{\min }(P)}} \sqrt{\frac{1}{3 c_{3}}\left(\left(c_{3} T+2 c_{1}\right)^{3}-8 c_{1}^{3}\right)} .
$$

\section{REFERENCES}

[1] G. Zames and N. A. Shneydor, "Dither in nonlinear systems," IEEE Trans. Automat. Contr., vol. 21, pp. 660-667, Oct. 1976.

[2] — - "Structural stabilization and quenching by dither in nonlinear systems," IEEE Trans. Automat. Contr., vol. 22, pp. 352-361, June 1977.

[3] S. Mossaheb, "Application of a method of averaging to the study of dither in nonlinear systems," Int. J. Contr., vol. 38, no. 3, pp. 557-576, 1983.

[4] K. J. Åström and B. Wittenmark, Adaptive Control. Reading, MA: Addison-Wesley, 1989. 
[5] B. Armstrong-Helouvry, Control of Machines with Friction. Boston, MA: Kluwer, 1991.

[6] B. Armstrong-Helouvry, P. Dupont, and C. C. de Wit, "A survey of models, analysis tools and compensation methods for control of machines with friction," Automatica, vol. 30, no. 7, pp. 1083-1138, 1994.

[7] B. Lehman and R. Bass, "Extensions of averaging theory for power electronic systems," IEEE Trans. Power Electron., vol. 11, pp. 542-553, July 1996.

[8] A. V. Peterchev and S. R. Sanders, "Quantization resolution and limit cycling in digitally controlled PWM converters," Proc. 32nd IEEE Power Electronics Specialists Conf., vol. 2, pp. 465-471, June 2001.

[9] R. M. Gray and D. L. Neuhoff, "Quantization," IEEE Trans. Inform. Theory, vol. 44, pp. 2325-2383, Oct. 1998.

[10] Y. Z. Tsypkin, Relay Control Systems. Cambridge, U.K.: Cambridge Univ. Press, 1984

[11] V. I. Utkin, Sliding Modes in Control and Optimization. Berlin, Germany: Springer Verlag, 1992.

[12] A. A. Pervozvanski and C. C. de Wit, "Asymptotic analysis of the dither effect in systems with friction," Automatica, vol. 38, no. 1, pp. 105-113, 2002.

[13] D. Atherton, Nonlinear Control Engineering. $\quad$ London, U.K.: Van Nostrand Reinhold, 1975.

[14] A. Gelb and W. Vander-Velde, Multiple Input Describing Functions. New York: McGraw-Hill, 1968.

[15] A. K. Gelig and A. Churilov, Stability and Oscillations of Nonlinear Pulse Modulated Systems. Berlin, Germany: Birkhäuser, 1998.

[16] A. A. Andronov, S. E. Khaikin, and A. A. Vitt, Theory of Oscillators. Oxford, U.K.: Pergamon Press, 1965.

[17] K. J. Åström and T. Hägglund, PID Controllers: Theory, Design, and Tuning, 2nd ed. Research Triangle Park, NC: Instrument Society of America, 1995

[18] N. Elia and S. K. Mitter, "Stabilization of linear systems with limited information," IEEE Trans. Automat. Control, vol. 46, pp. 1384-1400, Sept. 2001.

[19] A. S. Morse, "Control using logic-based switching," in Trends in Control. A European Perspective, A. Isidori, Ed. New York: Springer, 1995, pp. 69-113.

[20] K. J. Åström, "Oscillations in systems with relay feedback," in Adaptive Control, Filtering, and Signal Processing, K. J. Åström, G. C. Goodwin, and P. R. Kumar, Eds. New York: Springer-Verlag, 1995, vol. 74, IMA Volumes in Mathematics and its Applications, pp. 1-25.

[21] K. H. Johansson, A. Rantzer, and K. J. Åström, "Fast switches in relay feedback systems," Automatica, vol. 35, no. 4, pp. 539-552, 1999.

[22] M. di Bernardo, K. H. Johansson, and F. Vasca, "Self-oscillations and sliding in relay feedback systems: Symmetry and bifurcations," Int. J. Bifurcation Chaos, vol. 11, no. 4, pp. 1121-1140, 2000.

[23] J. M. Goncalves, A. Megretski, and M. A. Dahleh, "Global stability of relay feedback systems," IEEE Trans. Automat. Contr, vol. 46, pp. 550-562, Apr. 2001.

[24] S. Varigonda and T. T. Georgiou, "Dynamics of relay relaxation oscillators," IEEE Trans. Automat. Contr., vol. 46, pp. 65-77, Jan. 2001.

[25] K. H. Johansson, A. Barabanov, and K. J. Atröm, "Limit cycles with chattering in relay feedback systems," IEEE Trans. Automat. Contr., vol. 47, pp. 1414-1423, Sept. 2002.

[26] P. A. Cook, "Simple feedback systems with chaotic behavior," Syst. Contr. Lett., vol. 6, pp. 223-227, 1985.

[27] L. Iannelli, "Dither for Smoothing Relay Feedback Systems: An Averaging Approach,” Ph.D, University of Napoli Federico II, Naples, Italy, 2002.

[28] G. Zames and P. Falb, "Stability conditions for systems with monotone and slope-restricted nonlinearities," SIAM J. Contr., vol. 6, no. 1, pp. 89-108, 1968.

[29] A. Megretski and A. Rantzer, "System analysis via integral quadratic constraints," IEEE Trans. Automat. Contr., vol. 42, pp. 819-830, June 1997.

[30] L. Iannelli, K. H. Johansson, U. Jönsson, and F. Vasca, "Practical stability and limit cycles of dithered relay feedback systems," in Proc. Eur. Control Conf. 03, Cambridge, U.K., Sept. 2003.

[31] —_ "Effects of dither shapes in nonsmooth feedback systems: Experimental results and theoretical insight," in Proc. 42nd IEEE Conf. Decision and Control, Maui, HI, Dec. 2003.

[32] S. Sastry, Nonlinear Systems: Analysis, Stability and Control. New York: Springer-Verlag, 1999

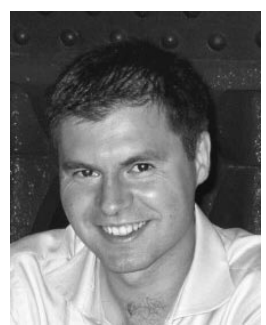

Luigi Iannelli (S'01-M'03) was born in 1975 in Benevento, Italy. He received the Master's (Laurea) degree in computer engineering from the University of Sannio,Benevento, Italy, in 1999, and the Ph.D. degree in information engineering from the University of Napoli "Federico II", Naples, Italy in 2003.

He was a Researcher for one semester in the Department of Signals, Sensors and Systems, Royal Institute of Technology (KTH), Stockholm, Sweden, while pursuing his doctoral degree, where he is now a Guest Researcher.His current research interests include analysis and control of switched and hybrid systems, automotive control and applications of control theory to electronic systems.

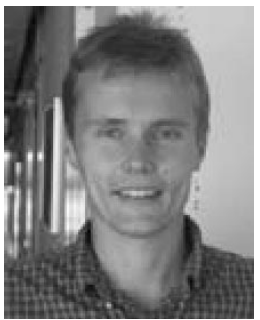

Karl Henrik Johansson (S'92-M'98) received the M.S. and Ph.D. degrees in electrical engineering, from Lund University, Lund, Sweden, in 1992 and 1997, respectively.

He held positions as an Assistant Professor at Lund University (1997-1998) and as a Visiting Research Fellow at the University of California, Berkeley (1998-2000). Currently, he is an Associate Professor in the Department of Signals, Sensors and Systems at the Royal Institute of Technology, Stockholm, Sweden. His research interests are in hybrid and switched systems, distributed embedded control, and applications in communication networks and automotive vehicles.

Dr. Johansson received the Young Author Prize of the IFAC World Congress in 1996, the Peccei Award from IIASA, Austria, in 1993, and a Young Researcher Award from Scania, Sweden, in 1996.

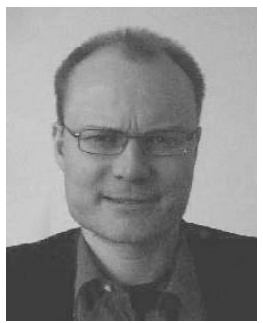

Ulf T. Jönsson (M'03) was born in Barsebäck, Sweden. He received the Ph.D. degree in automatic control from the Lund Institute of Technology, Lund, Sweden, in 1996.

He was a Post-Doctoral Fellow at the California Institute of Technology, Pasadena, and at the Laboratory for Information and Decision Systems, Massachusetts Institute of Technology, Cambridge. $\mathrm{He}$ is now an Associate Professor in the Division of Optimization and Systems Theory, Royal Institute of Technology. His current research interests include design and analysis of nonlinear and uncertain hybrid systems, periodic system theory, and convex optimization applications in systems theory.

Dr. Jönsson is an Associate Editor for the IEEE TRANSACTIONS ON AUTOMATIC CONTROL.

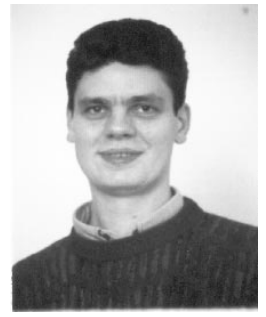

Francesco Vasca (S'94-M'95) was born in Giugliano, Naples, Italy, in 1967. He received the "Laurea" degree in electronic engineering and the Ph.D. degree in automatic control from the Università di Napoli Federico II, Naples, Italy, in 1991 and 1995, respectively.

Since 2000, he has been an Associate Professor of Automatic Control with the Dipartimento di Ingegneria, Università del Sannio, Benevento, Italy. His research interests include control and parameter identification for induction motors, nonlinear dynamics and control techniques for dc/dc converters, Kalman filtering for bilinear systems, analysis of feedback relay systems, control techniques for hybrid vehicles, modeling, and control of automated manual transmissions. 